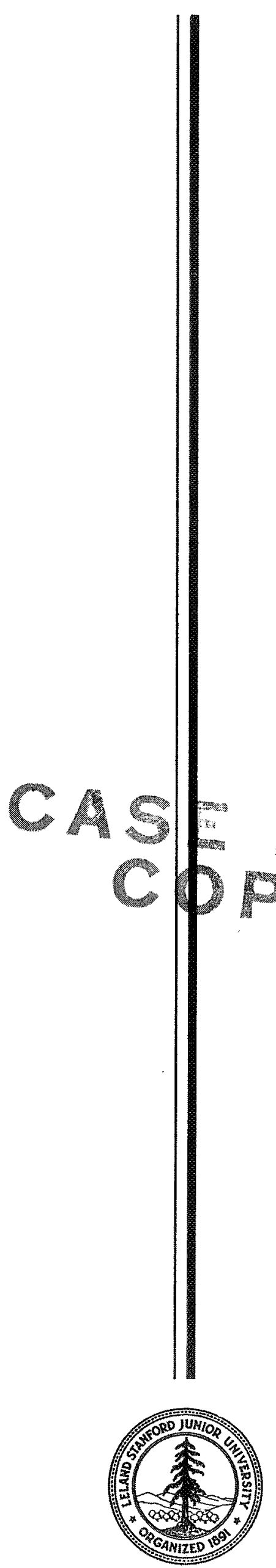

$$
\begin{array}{r}
N 71-19294 \\
\text { NASA CR }-116872
\end{array}
$$

\title{
Structure of the Chromosphere-Corona Transition Region
}

\author{
by \\ R. L. Moore and P. C. W. Fung \\ January 1971
}

SUIPR Report No. 377

\author{
Prepared under \\ National Aeronautics and Space Administration \\ Grant NGL 05-020-272 \\ and \\ Air Force Office of Scientific Research \\ Contract F44620-69-C-0008
}

\author{
INSTITUTE FOR PLASMA RESEARCH \\ STANFORD UNIVERSITY, STANFORD, CALIFORNIA
}




\title{
STRUCTURE OF THE CHROMOSPHERE-CORONA TRANSITION REGION
}

by

R. L. Moore and P.C.W. Fung

January 1971

\author{
National Aeronautics and Space Administration Grant NGL 05-020-272 \\ and \\ Air Force Office of Scientific Research \\ Contract F44620-69-C-0008
}

SUIPR Report No. 377

\author{
Institute for Plasma Research \\ Stanford University \\ Stanford, California
}




\begin{abstract}
The structure and energy balance of the chromosphere-corona transition region is investigated by means of a static, planar model which is compared with the results of XUV-resonance-line observations. In this model, the transition region is heated by thermal conduction from the corona and cooled by radiative losses. Comparison of the model with observational results implies that this is the dominant process in the energy balance of the transition region, and that the base of the transition region is inherently non-static and/or non-planar. The model explains the observational finding of Noyes et. al. (1970) that the number density and the downward heat flux both increase by the same factor from quiet regions to active regions. The implications of these results are discussed with regard to spicules.
\end{abstract}




\section{INTRODUCTION}

The purpose of this article is to examine the extent to which a static, planar model may be used to describe and explain the structure and energy balance of the chromosphere-corona transition region. In this section we shall briefly present the salient features of the transition region which motivate such a study.

In this paper, following Pottasch (1964), we shall use the term "chromosphere-corona transition region", or just "transition region", to designate the region of the solar atmosphere in which the temperature increases from about $10^{4} \mathrm{~K}$ (typical temperature of the upper chromosphere) to about $10^{6} \mathrm{~K}$ (typical temperature of the lower corona). This is the region from about $2,000 \mathrm{~km}$ to about $10,000 \mathrm{~km}$ above the photosphere (Athay, 1969, Dupree and Goldberg, 1967). Observed energy fluxes of extreme ultraviolet resonance lines emitted from the outer solar atmosphere imply (see Section 3.2.1) that above the $10^{5} \mathrm{~K}$ level the atmosphere is approximately planar, and that the flux of heat flowing from the corona down to the chromosphere remains roughly constant from the $10^{6} \mathrm{~K}$ level down to the $10^{5} \mathrm{~K}$ level. We term this upper part of the transition region the "constant-heat-flux region." The part of the transition region below the constant-heat-flux region will be called the "base region". This schematic picture of the transition region is summarized in Figure 1 .

A major function of the base region is to absorb the downward flowing heat which passes through the constant-heat-flux region. The value of this heat flux which enters the base region is not accurately determined by the ultraviolet line data, but a value greater than $10^{5}$ erg $\mathrm{cm}^{-2} \mathrm{sec}^{-1}$ is indicated (see section 3.2.1). At the $10^{4} \mathrm{~K}$ level and 
below, the heat conductivity and the temperature gradient are so small that less than $10^{2} \mathrm{erg} \mathrm{cm}^{-2} \mathrm{sec}^{-1}$ can be conducted out of the bottom of the transition region. Therefore, the heat entering the base region from the constant-heat-flux region must be absorbed in the base region. Optical eclipse spectra indicate that spicule-like inhomogeneities begin to appear in the chromosphere at heights above about $1,500 \mathrm{~km}$ (Suemoto and Hiei, 1962), and spicules often extend to heights of 10,000 $\mathrm{km}$ or more. Thus, some spicules extend through the height range of the chromosphere-corona transition region. Since spicules are transient and must have a temperature well below $10^{5} \mathrm{~K}$ (Beckers, 1968), the constantheat-flux region cannot be completely static and horizontally uniform. However, above $3,000 \mathrm{~km}$ spicules occupy less than a few percent of the horizontal surface area (Allen, 1963, Beckers, 1968). This suggests that the static, planar constant-heat-flux region implied by the ultraviolet emission-line data corresponds to a hot, static background atmosphere which is penetrated here and there by the cooler spicules. Thus, although the constant-heat-flux region appears to be inhomogeneous and fluctuating when viewed optically at the limb in a chromospheric emission lines, in terms of overall structure and average heat flow from the corona to the chromosphere, a static, planar model is still reasonable.

In view of the static, planar nature of the constant-heat-flux region (despite the presence of spicules), it is reasonable to consider a static, planar model for the base region which absorbs the heat which passes through the constant-heat-flux region. A static, planar model of the base region is "possible" if the downward heat flux passing the 
$10^{5} \mathrm{~K}$ level can be radiated away above the $10^{4} \mathrm{~K}$ level. However, Kuperus and Athay (1967) have suggested that the base region is so thin that the inflowing heat cannot be balanced by radiation alone, and that the excess energy goes into the kinetic energy of spicules. Hence, a quantitative study of a static, planar model of the transition region may be relevant to the origin of spicules, and their role and importance in the energy balance of the base region. 


\section{MODEL AND FORMULATION}

\subsection{BASIC PHYSICAL ASSUMPTIONS}

The model of the transition region studied in this paper is based on the following assumed physical conditions and approximations.

(1) The model is horizontally inform.

(2) The magnetic field is assumed to be vertical.

(3) The model transition region is assumed to be in hydrostatic equilibrium.

(4) The "turbulent pressure" in the transition region, due to velocity fluctuations (part of which are produced by the mechanical waves which pass through the transition region to heat the corona), is neglected; the model atmosphere is assumed to be supported against gravity entirely by the thermal gas pressure.

(5) There is no dissipation of the mechanical waves within the model transition region.

(6) The condition of ionization equilibrium is satisfied at each point in the model.

(7) All excitations and ionizations are collisional, and all recombinations and de-excitations are radiative.

(8) The presence of elements other than hydrogen is ignored except for their contribution to the radiative losses. The aim of each of the above adopted conditions is to simplify the mathematical model while hopefully retaining the essential physics involved in the structure and energy balance of the actual transition region. In particular, approximation (4) simplifies the force equation, 
approximation (5) simplifies the energy equation, while approximations (7) and (8) simplify the ionization equation as well as the force and energy equations. The degree to which the model resulting from our simplifying assumptions retains the essential physics of the transition region is to be judged, in part, from the comparison of the model with observations.

It should be noted that approximations (5) and (7) optimize the removal of heat $f 1$ ux by radiation. In general, the energy radiated from each volume element of the atmosphere may come from three sources: (i) mechanical energy dissipated in the volume, (ii) radiation absorbed in the volume, (iii) heat flux absorbed in the volume. Approximations (5) and (7) require that all of the energy radiated from each volume element be supplied by the absorption of heat flux.

\subsection{GOVERNING EQUATIONS}

The equation which expresses condition (6), the condition of ionization equilibrium, is

$$
\mathfrak{I}=\mathfrak{R},
$$

where $I$ is the rate of ionization per unit volume, and $R$ is the rate of recombination per unit volume. If the vertical coordinate $z$ is taken to be positive upward, the condition of hydrostatic equilibrium requires that

$$
\frac{d p}{d z}=-\rho g
$$

where $p$ is the thermal gas pressure, $\rho$ is the mass density of the gas and $\mathrm{g}$ is the acceleration of gravity. Conditions (3), (5), and (7) and the conservation of energy demand that there be a balance of 
heat conduction and radiation. This is expressed by

$$
-\frac{d F}{d z}=\mathfrak{L}_{r}
$$

where $F$ is the heat $f l u x$ (positive upward) and $\mathscr{L}_{r}$ is the radiative power output per unit volume. We now proceed to express equations (1), (2) and (3) in terms of the total number density $n$, the electron number density $\mathrm{n}_{e}$, the temperature $\mathrm{T}$, and their derivatives with respect to the vertical coordinate $z$, to obtain three equations in three unknowns with which to compute the structure of the model. Under approximations (7) and (8), equation (1) can be written as

$$
n_{e} n_{H^{T}}{ }=n_{e} n_{p} R_{H}
$$

where $n_{H}$ is the number density of neutral hydrogen, $n_{p}$ is the number density of ionized hydrogen, $I_{H}$ is the collisional ionization coefficient for hydrogen, and $\mathrm{R}_{H}$ is the radiative recombination coefficient for hydrogen. Now with the simple relations for pure hydrogen,

$$
n=n_{H}+n_{p}+n_{e}
$$

and

$$
\mathrm{n}_{\mathrm{p}}=\mathrm{n}_{\mathrm{e}}
$$

the ionization equilibrium equation may be solved for $n_{e} / n$ :

$$
\frac{\mathrm{n}_{\mathrm{e}}}{\mathrm{n}}=\frac{1}{2+\mathrm{R}_{\mathrm{H}} / \mathrm{I}_{\mathrm{H}}} \text {. }
$$


For pure hydrogen, the mass density of the gas is

$$
p=\left(1-\frac{n_{e}}{n}\right) n m_{H}
$$

From this expression and the equation of state,

$$
\mathrm{p}=\mathrm{nkT},
$$

the equation of hydrostatic equilibrium can be written in terms of $n$, $\mathrm{n} e$ and $\mathrm{T}$ :

$$
\frac{1}{n T} \frac{d(n T)}{d z}=-\frac{\left(1-n_{e} / n\right) m_{H} g}{k T} .
$$

The heat flux $F$ is proportional to the temperature gradient:

$$
\mathrm{F}=-\kappa \frac{\mathrm{dT}}{\mathrm{dz}}
$$

where $\kappa$ is the thermal conductivity. Thus, the left hand side of the energy equation, equation (3), may be expressed as

$$
-\frac{d F}{d z}=\frac{d}{d z}\left(\kappa \frac{d T}{d z}\right)
$$

For the radiative power output density $\mathcal{L}_{Y}$, we adopt the results of Cox and Tucker (1969). They have computed the radiative cooling coefficient $L_{r}$, which is related to $\mathcal{L}_{r}$ by

$$
\mathfrak{L}_{r}=\mathrm{n}_{\mathrm{e}}\left(\mathrm{n}_{\mathrm{H}}+\mathrm{n}_{\mathrm{p}}\right) L_{r}
$$

as a function of temperature in the range $10^{4} \mathrm{~K}$ to $10^{8} \mathrm{~K}$. In addition to hydrogen, Cox and Tucker have included cosmic abundances of the next eight most abundant elements: He, C, N, O, Ne, Mg, Si and S, in their calculation of $L_{r}$. At temperatures above a few times $10^{4} \mathrm{~K}$, 
these additional elements dominate the radiative cooling. Thus, with regard to $\mathrm{L}_{r}$, we relax our assumption of a pure hydrogen atmosphere. However, since hydrogen is the major component of the solar atmosphere, the elements other than hydrogen may be neglected in the equation of hydrostatic equilibrium, in determining the heat flux and thermal conductivity, and in the ionization equation, which determines the electron, proton and hydrogen number densities in equation (13). The expression of the energy equation in terms of $n, n_{e}$ and $T$ is then

$$
\frac{d}{d z}\left(\kappa \frac{d T}{d z}\right)=\frac{n_{e}}{n}\left(1-\frac{n_{e}}{n}\right) n^{2} L_{r} .
$$

\subsection{SPECIF ICATION OF THE MODEL}

The coefficients $I_{H}$ and $R_{H}$ in equation (7), and $k$ and $L_{r}$ in equation (14) are functions of temperature which we now specify.

The ionization and recombination coefficients for hydrogen are taken from Cox and Tucker (1969):

$$
I_{H}=2.34 \times 10^{-8} \beta^{-1 / 2} \mathrm{e}^{-\beta} \mathrm{cm}^{3} \mathrm{sec}^{-1}
$$

and

$$
R_{H}=5.20 \times 10^{-14} \beta^{1 / 2}\left[0.4288+\frac{1}{2} \ln \beta+0.4698 \beta^{-1 / 3}\right] \mathrm{cm}^{3} \mathrm{sec}^{-1},
$$

where

$$
\beta=158,000 / \mathrm{T} .
$$


Figure 2 shows the radiative cooling coefficient curve computed by Cox and Tucker for the temperature range $10^{4} \mathrm{~K}$ to $10^{6} \mathrm{~K}$. We have adopted the following straight-line-segment fit for our model:

$$
\left.\begin{array}{l}
4.0<\log \mathrm{T}<4.2: \log \mathrm{L}_{\mathrm{r}}=8.00 \log \mathrm{T}-55.6 \\
4.2<\log \mathrm{T}<4.5: \log \mathrm{L}_{\mathrm{r}}=-22.0 \\
4.5<\log \mathrm{T}<4.87: \log \mathrm{L}_{\mathrm{r}}=2.44 \log \mathrm{T}-33.0 \\
4.87<\log \mathrm{T}<5.5: \log \mathrm{L}_{\mathrm{r}}=-21.1 \\
5.5<\log \mathrm{T}<6.0: \log \mathrm{L}_{\mathrm{r}}=-1.86 \log \mathrm{T}-10.9
\end{array}\right\}
$$

Figure 3 shows the thermal conductivity curve which we have obtained from the results of Devoto (1968) and Delcroix and Lemaire (1969) (see Appendix A). The curve is closely fit by three straight line segments given by

$$
\left.\begin{array}{l}
4.0<\log T<4.05: \log \kappa=\log T+1.49 \\
4.05<\log \mathrm{T}<4.26: \log \kappa=-3.19 \log \mathrm{T}+18.47 \\
4.26<\log \mathrm{T}<6.00: \log \kappa=2.36 \log \mathrm{T}-5.18
\end{array}\right\}
$$

This straight-1ine-segment fit is the conductivity curve used in computing the structure of the model.

With the above coefficient formulas, we have now completely specified our model. The structure of the model is completely determined by appropriate boundary values of $\mathrm{n}, \mathrm{T}$, and $\mathrm{F}$. We choose to specify the values of these quantities at the upper boundary of the model transition region because the value of the number density is best known at the top of the observed transition region (Athay, 1969). We define the 
the upper boundary of the model to be the $10^{6} \mathrm{~K}$ level. We therefore fix the temperature $T_{O}$ of the upper boundary of the model at $10^{6} \mathrm{~K}$ and study the model by varying the boundary values of $n$ and $F$, which are denoted by $\mathrm{n}_{0}$ and $\mathrm{F}_{\mathrm{O}}$. 


\section{RESULTS}

\subsection{Numerical Results}

To study the behavior of the model with variations in $n_{O}$ and $F_{0}$, we computed the run of temperature and heat flux with height in the model for several values of $\mathrm{n}_{\mathrm{O}}$ and $\mathrm{F}_{\mathrm{O}}$ by numerically solving the governing equations (7), (10), and (14). Examples of the temperature profiles are shown in Figure 4, where $F_{0}$ is fixed at $-1.0 \times$ $10^{6} \mathrm{erg} \mathrm{\textrm {cm } ^ { - 2 }} \mathrm{sec}^{-1}$ and $\mathrm{n}_{0}$ takes the values $1.00,1.92,2.24$, and 2.82 times $10^{9} \mathrm{~cm}^{-3}$. Curves of the heat flux versus the logarithm of the temperature for the same cases are shown in Figure 5.

The curves in Figures 4 and 5 may be qualitatively understood as follows. The radiative power output density is proportional to $n^{2}$. On the other hand, if there were no radiative losses, the thickness of the transition region would be inversely proportional to the (constant) downward heat flux. Consequently, we expect that for sufficiently large values of the number density $n_{0}$ and sufficiently small values of the heat flux $F_{O}$ at the upper boundary, the model will radiate away all of the downward heat flux. That is, the heat flux will decrease to zero at some temperature $\mathrm{T}_{\mathrm{ZF}}>10^{4} \mathrm{~K}$. Figures 4 and 5 bear this out: if $n_{0}$ is large enough $\left(n_{0} \geqslant 1.92 \times 10^{9} \mathrm{~cm}^{-3}\right)$ the temperature gradient and the heat flux pass through zero at the minimum temperature $\left(>10^{4} \mathrm{~K}\right)$ reached by the curve, but if $\mathrm{n}_{0}$ is too small (e.g. $\mathrm{n}_{0}=1.00 \times 10^{9} \mathrm{~cm}^{-3}$ ), no temperature minimum occurs because the model is unable to radiate away all of the heat flux.

For a given $\mathrm{F}_{\mathrm{O}}$, the value of $\mathrm{n}_{\mathrm{O}}$ determines the temperature $\mathrm{T}_{\mathrm{ZF}}$ at which $F=0$. Thus, from sets of cases such as that represented in Figure 5, we may obtain the sets of $\left(n_{0}, T_{\mathrm{ZF}}\right)$ points shown in Figure 6 
for constant values of $\mathrm{F}_{\mathrm{O}}$. A smooth curve has been fitted through each set of points with a common value of $\mathrm{F}_{\mathrm{O}}$. From these curves we then obtain sets of $\left(\mathrm{n}_{\mathrm{O}}, \mathrm{F}_{\mathrm{O}}\right)$ at constant $\mathrm{T}_{\mathrm{ZF}}$ which we have plotted logarithmically in Figure 7. Each set of points for constant $T_{Z F}$ lies along a curve with slope increasing from about 0.9 at $\log n_{0}=9.0$ to about 1.0 at $\log \mathrm{n}_{\mathrm{O}}=10.0$. Thus, for constant $\mathrm{T}_{\mathrm{ZF}}, \mathrm{F}_{\mathrm{O}}$ is approximately proportional to $\mathrm{n}_{\mathrm{O}}$.

The fact that $F_{O}$ is approximately proportional to $n_{0}$ for constant $T_{Z F}$ is readily understood from the governing equations and the fact that the pressure is nearly constant in the model transition region. With the aid of the heat conduction law, equation (11), the energy equation (14) may be written as

$$
F d F=\left[\frac{n_{e}}{n}\left(1-\frac{n_{e}}{n}\right) \frac{L_{r}}{T^{2}} \kappa\right](n T)^{2} d T
$$

Equation (7) shows that the quantity in square brackets is a function of the temperature alone:

$$
\frac{\mathrm{n}_{\mathrm{e}}}{\mathrm{n}}\left(1-\frac{\mathrm{n}_{\mathrm{e}}}{\mathrm{n}}\right) \frac{\mathrm{L}_{r}}{\mathrm{~T}^{2}} \kappa \equiv f(T) .
$$

In conjunction with the heat conduction law, the equation of hydrostatic equilibrium yields the integral

$$
n T=n_{O} T_{O} \exp \left[-\int_{T}^{T_{O}} \frac{\kappa}{H F} d T\right]
$$


where $H$ is the pressure scale height,

$$
H=\frac{k T}{\left(1-\frac{n}{n}\right) m_{H} g}
$$

The quantity $-\int_{T}^{T_{O}} k / H F d T$ is positive due to the fact that $F$ is negative (heat flux downward). Even though $F$ goes to zero, so that - $\frac{K}{\mathrm{HF}}$ goes to infinity, as $\mathrm{T}$ approaches $\mathrm{T}_{\mathrm{ZF}}$, it was found that

$$
-\int_{\mathrm{T}_{\mathrm{ZF}}}^{\mathrm{T}_{\mathrm{O}}} \frac{\kappa}{\mathrm{HF}} \mathrm{dT} \ll 1
$$

in all cases for which the model was computed. That is, in the ranges of $\mathrm{n}_{O}$ and $\mathrm{F}_{O}$ in Figure 6 , the pressure remains nearly constant through the model.

Equation (20) may therefore be written

$$
F d F \approx n_{O}^{2} T_{O}^{2} f(T) d T
$$

Integration of equation (25) from $\mathrm{T}_{\mathrm{ZF}}$ to $\mathrm{T}_{\mathrm{O}}$ gives

$$
\frac{1}{2} \dot{\mathrm{F}}_{0}^{2} \approx \mathrm{n}_{0}^{2} \mathrm{~T}_{0}^{2} \int_{\mathrm{T}_{\mathrm{ZF}}}^{\mathrm{T}_{\mathrm{O}}} f(\mathrm{~T}) \mathrm{dT}
$$

which shows that for constant $T_{O}$ and $T_{Z F}, F_{O}$ is approximately proportional to $\mathrm{n}_{0}$ 


\subsection{COMPARISON OF THE MODEL WITH XUV-RESONANCE-LINE DATA}

\subsubsection{Observational Evidence for a Planar Constant-Heat-F1ux Region}

The analyses of Athay (1966) and Dupree and Goldberg (1967) of the emission of XUV resonance lines from a planar transition region, in conjunction with the observed energy fluxes of several XUV resonance lines, provide the empirical evidence for a planar constant-heat-flux region in the temperature range $10^{5} \mathrm{~K}$ to $10^{6} \mathrm{~K}$. In these analyses the energy flux $E_{L}$ observed at the earth in an XUV resonance line is related to the structure of the transition region by

$$
E_{L}=C_{L} A P_{e^{2}}^{-1}
$$

Here $P_{e}$ and $G$ are "representative" values of the electron pressure divided by Boltzmann's constant $\left(P_{e} \equiv n_{e} T\right)$ and of the temperature gradient, respectively, in the layer of the transition region in which the line is formed. A is the abundance (element:hydrogen ratio) of the element which emits the line. $C_{L}$ is a constant of proportionality, which is evaluated for each line by integrating a function of temperature (proportional to the number density of the ion which emits the line and the rate of excitation of the line) over the temperature range in which the ion is produced. This integrand is a sharply peaked function of temperature, contributing significantly to $C_{L}$ only at temperatures within about a factor of two or less from the temperature $T_{\max , L}$ at which it is maximum. Hence, equation (27) may be used to obtain an estimate of $A\left(n_{e} T\right)^{2}\left(\frac{d T}{d z}\right)^{-1}$ at $T_{\max , L}$ from the observed energy flux $F_{L}$. 
The data points in Figure 8 (cf. Figure 3 of Dupree and Goldberg) were obtained in this way from the values of $\mathrm{C}_{\mathrm{L}}$ and $\mathrm{T}_{\max , \mathrm{L}}$ computed by Dupree and Goldberg. We used the observed values of $E_{L}$ adopted by them for several XUV resonance lines emitted from oxygen ions and silicon ions formed in the temperature range of the transition region. The value of $(\mathrm{nT})^{2}\left(\frac{\mathrm{dT}}{\mathrm{dz}}\right)^{-1}$ for each data point was obtained by assuming that the abundances of oxygen and silicon are the photospheric abundances of Goldberg et al. (1960), $A_{O}=9.3 \times 10^{-4}, A_{{ }_{S i}}=3.2 \times 10^{-5}$, and by taking $\mathrm{n}=2 \mathrm{n} \mathrm{e}$. Since the pressure remains nearly constant, the straight lines of slope 2.36 in Figure 8 correspond to constant downward heat fluxes between $10^{5} \mathrm{~K}$ and $10^{6} \mathrm{~K}$, since above $10^{5} \mathrm{~K}$ the heat conductivity is proportional to $\mathrm{T}^{2.36}$. The solid line is the best visual fit to the data in the range $10^{5} \mathrm{~K}$ to $10^{6} \mathrm{~K}$, while the dashed lines show that the data scatter about the solid line by a factor of two. The data thus imply that, between $10^{5} \mathrm{~K}$ and $10^{6} \mathrm{~K}$, the transition region is approximately planar, and that in this region the heat flux varies by less than a factor of four. We see that although the heat flux need not stay completely constant in the constant-heat-flux region, a sizable fraction (25\% or more) of the heat flux at the top of the transition region should reach the $10^{5} \mathrm{~K}$ level.

\subsubsection{Deduction of Values of $n_{0}$ and $F_{0}$ from the Line Data}

The energy fluxes analyzed by Dupree and Goldberg were composed of radiation from all areas of the observable solar hemisphere, including some active regions. However, Withbroe (1970) concludes from OSO-IV XUV-resonance-line observations (with spatial resolution of one minute of arc) that "... for XUV lines formed at temperatures less than about 
$10^{6} \mathrm{~K}$ the flux radiated by the entire solar disk is characteristic of the equatorial quiet area." On this basis we assume that at $10^{6} \mathrm{~K}$ and below the data in Figure 8 represent the quiet solar atmosphere. We then obtain from Figure 8 (at $T=T_{O}=10^{6} \mathrm{~K}$ ) the following constraint on $n_{0}$ and $F_{0}$ for quiet regions:

$$
\log n_{0}=\frac{1}{2} \log \left(-F_{0}\right)+6.06 \pm 0.15
$$

Withbroe (1970) has studied the limb brightening of several XUV lines observed by OSO-IV in the quiet equatorial region. He finds good agreement between the observed limb brightening and that computed for a planar model transition region and lower corona having constant downward heat flux in the region below the $2 \times 10^{6} \mathrm{~K}$ level. Withroe's analysis of the limb brightening data allows an estimate of the downward heat flux independent of the number density. Withbroe finds

$$
\log \frac{\alpha}{(-F)}=-12.0 \pm 0.3
$$

where $F$ is the constant downward heat flux and $\alpha$ is the coefficient in the thermal conductivity used in his model:

$$
k=\alpha T^{5 / 2}
$$

For our adopted thermal conductivity, $\alpha$ has the value $1.0 \times 10^{-6}$ (cgs units) at the $10^{6} \mathrm{~K}$ temperature of the upper boundary. Using this value in equation (29), we obtain the value of $F_{0}$ which we adopt for the quiet regions:

$$
\log \left(-F_{0}\right)=6.0 \pm 0.3
$$


Equations (28) and (31) define the $\left(n_{0}, F_{0}\right)$ region required by the data for quiet regions. From equation $(28)$, for $\log \left(-F_{0}\right)=6.0$,

$$
\log n_{0}=9.06 \pm 0.15
$$

which is a reasonable value for the number density at the base of the quiet corona.

Using the model of Withbroe (1970), Noyes et al. (1970) we have compared the observed XUV resonance Iine emission from quiet regions with that from active regions. They find that, for a fixed temperature, the total number density and the downward heat flux in active regions are each about five times larger than in quiet regions. For active regions we therefore adopt, in place of equations (28) and (31),

$$
\log n_{0}=\frac{1}{2} \log \left(-F_{0}\right)+6.76 \pm 0.15
$$

and

$$
\log \left(-F_{0}\right)=6.7 \pm 0.3
$$

In Figure 9 , the two $\left(\log n_{O}, \log F_{0}\right)$ regions adopted above for quiet regions and active regions of the sun are compared with the lines of constant $\mathrm{T}_{\mathrm{ZF}}$ for the model. The data of Figure 8 require that $\mathrm{T}_{\mathrm{ZF}}<1 \times 10^{5} \mathrm{~K}$ in quiet regions, and the results of Noyes et a1. imply that $\mathrm{T}_{\mathrm{ZF}}<2 \times 10^{5} \mathrm{~K}$ in active regions. Hence, in the shaded areas of Figure 9, the model is compatible with the XUV-resonanceline observations. 


\section{DISCUSSION}

Given that $\mathrm{T}_{\mathrm{ZF}}<2 \times 10^{5} \mathrm{~K}$ in both quiet regions and active regions, our model yields the observational finding that $\mathrm{F}_{O}$ and $\mathrm{n}_{O}$ increase by the same factor from quiet regions to active regions. Moreoever, although there is some discrepancy, the model is compatible with the magnitudes of $F_{O}$ and $n_{O}$ required by the XUV observations of quiet regions and active regions. These two favorable results imply that, in a first approximation, the model is a valid description of the structure and the energy balance of the transition region. That is, these results imply that the dominant process in the energy balance of the transition region is the removal of heat flux by radiation, and that, with respect to the overall balance of energy, the transition region may be considered to be static and planar. The fact that the model agrees with both quiet regions and active regions implies that when the downward heat flux increases, the density of the atmosphere automatically increases enough to radiate away the larger heat flux.

The preceding paragraph interprets the agreement between the model and the data. It is also instructive to consider the disagreement between the model and the data. It was found in section 3.2.1 that the data require that $25 \%$ or more of the heat flux which flows into the transition region from the corona be absorbed in the base region. But, as can be seen in Figure 5, the model base region is capable of absorbing only about $20 \%$ or less of the heat flux $F_{O}$ which enters the model transition region. This is the cause of the discrepancy between the model and the data in Figure 9 . For there would be much better agreement 
between the model and the data in Figure 9 if, for $-\mathrm{F}_{0}=1.0 \times 10^{6} \mathrm{erg}$ $\mathrm{cm}^{-2} \mathrm{sec}^{-1}, \mathrm{~T}_{\mathrm{ZF}}=10^{4}$ were given by $\mathrm{n}_{0} \simeq 1.0 \times 10^{9} \mathrm{~cm}^{-3}$ instead of by $n_{0} \simeq 2.0 \times 10^{9} \mathrm{~cm}^{-3}$. In Figure 5, we see that this would occur. if about $40 \%$ (instead of $20 \%$ ) of $\mathrm{F}_{\mathrm{O}}$ were absorbed by the model base region. Thus, we conclude that the discrepancy between the model and the data in Figure 9, implies that the base region of the model absorbs only about half as much heat flux as the base region of the actual transition region.

We expect the actual base region to radiate away more heat than the planar model base region (see Appendix B). In Figure 4, we see that for $\mathrm{T}_{\mathrm{ZF}}=1.5 \times 10^{4} \mathrm{~K}$, the layer between $10^{5} \mathrm{~K}$ and $\mathrm{T}_{\mathrm{ZF}}$ is only 10 or $20 \mathrm{~km}$ thick. One effect of oscillations (of scale $\approx 10^{3} \mathrm{~km}$ ) in the base region, due in part to the passage of the compression waves which heat the corona, should be to increase the surface area of the thin layer between $10^{5} \mathrm{~K}$ and $10^{4} \mathrm{~K}$ over that of the planar model, thus making the base of the transition region a more efficient radiator than if it were strictly planar. Another effect of the velocity fluctuations is to increase the number density in the base region, which increases the radiative power output density. A factor of two increase in the amount of heat flux radiated away in the base region by these two mechanisms is quite plausible (see Appendix B). These considerations suggest, first, that the dominant process in the energy balance of the base region is the removal of heat flux by radiation and, second, that with respect to the energy balance, the base region is inherently non-static and/or non-planar.

The preceding discussion suggests that the energy lost from the 
base of the transition region in the form of spicule kinetic energy is secondary to that lost by radiation. However, the spicule kinetic energy may still be derived from the downward heat flux as suggested by Kuperus and Athay (1967). If, for a given heat flux $\mathrm{F}_{\mathrm{O}}$, the number density $n_{0}$ is too low, the point $\left(n_{0}, F_{0}\right)$ in Figure 9 will lie to the left of the $\mathrm{T}_{\mathrm{ZF}}=10^{4} \mathrm{~K}$ line where the model is unable to radiate away all of $F_{O}$. In this case, we expect the atmosphere to react by increasing $n_{O}$ until $F_{O}$ can be radiated away. This suggests that the excess heat flux would go into raising material at the base of the transition region to higher levels. Spicules occur over the boundaries of the supergranulation cells where the convective motion of the cells has concentrated the magnetic field. The magnetic field fans out above the boundaries and funnels the downward flowing heat into regions over the cell boundaries, giving the supergranule boundary region heat flux $F_{O}$ which is larger than the average value for quiet regions (Kopp and Kuperus, 1968). This suggests that spicules are the manifestation of the atmosphere attempting to maintain an increased density over the supergranule boundary regions. The transient character of spicules suggests an overstable situation in which the density is altemately higher or lower than the value required to balance the inflowing heat with radiative losses. 


\section{APPENDIX A: THERMAL CONDUCTIVITY IN THE TRANSITION REGION}

For the purposes of our model, we need an estimate of the thermal conductivity as a function of temperature which differs from the actual conductivity by less than a factor of two. The temperature range of the transition region is from $10^{4} \mathrm{~K}$, where hydrogen is nearly completely neutral, to $10^{6} \mathrm{~K}$, where hydrogen is essentially completely ionized. Since the solar atmosphere is composed of about $90 \%$ hydrogen by number, the thermal conductivity of the transition region is approximated with sufficient accuracy by the thermal conductivity of partially ionized hydrogen in the temperature range from $10^{4} \mathrm{~K}$ to $10^{6} \mathrm{~K}$.

It was assumed, in computing the radiative energy losses and the degree of ionization in the model, that all excitations and ionizations of the hydrogen atoms are collisional, and all recombinations and deexcitations are radiative. We also assume this condition in estimating the thermal conductivity. Under this condition, only the thermal kinetic energy of the electrons, protons, and hydrogen atoms contributes to the heat conduction; the energy of excitation and ionization of the hydrogen atoms does not contribute.

The thermal conductivity of partially ionized hydrogen due to the transport of thermal kinetic energy has been computed by Devoto (1968) from accurate but rather complicated kinetic theory. It appears that his computed values should approximate the actual conductivity with an accuracy of the order of 10\%. The curves of Figure 10 have been obtained from Devoto"s table of the conductivity as a function of temperature at constant pressure of $10^{4}, 10^{5}$, and $10^{7}$ times 1.013 dyne $\mathrm{cm}^{-2}$ $\left(1.013\right.$ dyne $\left.\mathrm{cm}^{-2}=10^{-6} \mathrm{~atm}\right)$. None of these curves can be taken for 
the thermal conductivity of the transition region for the following two reasons. First, the conductivity computed by Devoto obviously depends on the pressure, and the pressures adopted in Devoto's computations are much larger than the pressure of about 0.2 dyne $\mathrm{cm}^{-2}$ (A.thay, 1969) in the transition region. Second, the conductivity depends on the degree. of ionization. In computing the degree of ionization, Devoto assumed local thermodynamic equilibrium. In this case, the degree of ionization depends on the pressure; an increase of pressure at constant temperature causes a decrease in the degree of ionization. But in the transition region, under the condition of collisional excitation and ionization, and radiative recombination and de-excitation, the plasma is not in local thermodynamic equilibrium; the degree of ionization depends only on the temperature and not on the pressure (see equations (7), (15) and (16)). Thus, we would not be justified in adopting Devoto's published values of the thermal conductivity of partially ionized hydrogen for the thermal conductivity of the transition region.

However, instead of repeating Devoto's involved and lengthy calculations for the case of the transition region, we can estimate the conductivity of the transition region with sufficient accuracy from Devoto's values by deriving from simple kinetic theory the manner in which the conductivity scales with pressure and degree of ionization. Using simple kinetic theory (Vincinti and Kruger, 1965), we can derive the following formula for the thermal conductivity of partially ionized hydrogen:

$$
\kappa=\frac{9}{4} \frac{\mathrm{k}^{3 / 2}}{\mathrm{~m}_{\mathrm{H}}^{1 / 2}} \frac{\mathrm{T}^{1 / 2}}{\left(\sigma_{\mathrm{HH}}+\mathrm{n}_{\mathrm{p}} / \mathrm{n}_{\mathrm{H}} \sigma_{\mathrm{Hp}}\right)}+\kappa_{\mathrm{e}} \cdot
$$


Here $\sigma_{\mathrm{HH}}$ and $\sigma_{\mathrm{Hp}}$ are "collision cross-sections" for energy exchange between neutral hydrogen atoms, and between neutral hydrogen atoms and protons, respectively. Since $\sigma_{\mathrm{HH}}$ and $\sigma_{\mathrm{Hp}}$ are of the nature of crosssections for binary collisions, we expect them to depend on the temperature but not on the number densities. $k_{e}$ in equation (35) is the thermal conductivity of fully ionized hydrogen, which may be computed from the formula given by Delcroix and Lemaire (1969):

$$
\kappa_{e}=1.890 \times 10^{-5} \frac{\mathrm{T}^{5 / 2}}{\ln \Lambda} \operatorname{erg} \mathrm{sec}^{-1} \mathrm{~K}^{-1} \mathrm{~cm}^{-1}
$$

where

$$
\left.\begin{array}{ll}
\Lambda=\frac{3}{\sqrt{2 \pi}} \frac{k^{2}}{e^{3}} \frac{T^{2}}{p^{1 / 2}} & \text { for } T<4.2 \times 10^{5} \mathrm{~K} \\
\Lambda=\frac{3}{\sqrt{2 \pi}} \frac{k^{2}}{e^{3}} \frac{T^{2}}{p^{1 / 2}}\left(\frac{4.2 \times 10^{5}}{T}\right)^{1 / 2} & \text { for } T>4.2 \times 10^{5} \mathrm{~K} .
\end{array}\right\}
$$

Here $e$ is the electron charge is esu and $p$ is the pressure. Equation (35), in conjunction with equations (36) and (37), gives explicit1y the dependence of the conductivity on the ion-neutral ratio $n_{p} / n_{H}$ and on the pressure p. We use this property of equation (35) to estimate the conductivity of the transition region from Devoto's results shown in Figure 10.

The estimate of the conductivity in the transition region is obtained as follows. First, Devoto's results are used to determine functions of temperature representing $\sigma_{\mathrm{HH}}$ and $\sigma_{\mathrm{Hp}}$ such that equation (35) approximately reproduces Devoto's conductivity. This is done by fitting equation (35) to the curves in Figure 10, using Devoto's values of 
$n_{p} / n_{H}$ and $p$. The good fit shown in Figure 11 is given by

$$
\sigma_{\mathrm{HH}}=9.12 \times 10^{-14} \mathrm{~T}^{-1 / 2} \mathrm{~cm}^{2}
$$

and

$$
\sigma_{\text {Hp }}=7.95 \times 10^{-11} \mathrm{~T}^{-1} \mathrm{~cm}^{2} \text {. }
$$

With these functions of temperature for $\sigma_{\mathrm{HH}}$ and $\sigma_{\mathrm{Hp}}$, equation (35) reproduces Devoto's conductivity with an error of not more than about 10\%. The thermal conductivity for the transition region is then computed from equation ( 35 ), keeping these functions of temperature for $\sigma_{\mathrm{HH}}$ and $\sigma_{\mathrm{Hp}}$, but using values of $\mathrm{n}_{\mathrm{p}} / \mathrm{n}_{\mathrm{H}}$ and $\mathrm{p}$ appropriate for the transition region. We assumed 0.2 dyne $\mathrm{cm}^{-2}$ for $\mathrm{p}$, and $\mathrm{n}_{\mathrm{p}} / \mathrm{n}_{\mathrm{H}}$ was calculated as a function of temperature from equations ( 7 ), (15), and (16). The resulting conductivity curve is shown in Figure 3 . 
There are three physically distinct energy fluxes which enter into the energy balance of the solar atmosphere: mechanical energy flux $\vec{F}_{m}$, radiative energy flux $\vec{F}_{r}$, and heat flux $\vec{F}_{c}$ due to thermal conduction. We assume that, on the average over a sufficiently large area and over a sufficiently long time, each of these fluxes is vertical and steady. In other words, we assume that on the scale of sufficiently large horizontal lengths and sufficiently long times, the structure and energy balance of the solar atmosphere may be considered to be horizontally uniform and steady in time. Each of the energy fluxes may vary with height in the atmosphere by depositing thermal energy in the atmosphere or removing thermal energy from the atmosphere. The general form of the steady-state energy equation in terms of the energy fluxes is

$$
\frac{d F_{m}}{d z}+\frac{d F}{d z}+\frac{d F}{d z}=0
$$

In our model transition region we have assumed that $F_{m}$ is constant,

$$
\frac{\mathrm{dF}_{\mathrm{m}}}{\mathrm{dz}}=0
$$

and that there is no absorption of radiation,

$$
\frac{\mathrm{dF}}{\mathrm{dz}}>0
$$

Under these two conditions, the energy equation requires that the downward heat flux in the model transition region be absorbed by radiative losses. U1traviolet-resonance-line observations of the transition 
region indicate that the rate at which energy is supplied to the transition region by heat conduction from the corona and the rate at which energy is lost from the transition region by radiation are of the same order of magnitude $\left(10^{6} \mathrm{erg}^{-2} \mathrm{cec}^{-1}\right.$ ) (Osterbrock, 1961; Allen, 1965; Nikolsky, 1969). This is the observational basis for suspecting that the dominant processes in the energy balance of the transition region are the absorption of heat and radiative losses.

However, the comparison of our model with XUV-resonance-line data indicates that the actual base region absorbs about twice as much heat flux as the model base region (see section 4). This requires that

$$
\int_{\text {base }}\left(\frac{d F_{m}}{d z}+\frac{d F}{d z}\right) d z
$$

for the actual base region be larger than that for the model base region. An equivalent statement is that one or any combination of the following statements is true of the actual base region with respect to the model base region:

(1) The actual base region is thicker than the model base region, i.e. the volume of the actual base region is larger than that of the model base region.

(2) $\frac{\mathrm{dF}}{\mathrm{dz}}$ is larger in the actual base region than in the model base region, $i . e$. the radiative power output per unit volume is larger in the actual base region than in the model base region.

(3) $\frac{d F_{m}}{d z}$ is larger in the actual base region than in the model base region, i.e. $\frac{d F}{d z}>0$ in the actual base region.

Each of these statements names a possible mechanism by which the actual 
base region is able to absorb more heat flux than the model base region. The basic nature of the energy balance of the base region depends upon which of these mechanisms, if any, is dominant in the actual base region. If either of the first two mechanisms is dominant, the dominant process in the energy balance is the removal of heat flux by radiative losses as is assumed in the model. If the third mechanism is dominant, the dominant process in the energy balance is the conversion of thermal energy to mechanical energy.

The presence of the mechanical energy flux has been neglected in the model transition region. In the following paragraphs we estimate the effect of the mechanical energy flux on the structure of the base region. We find that the presence of the mechanical energy flux can reasonably increase the absorption of heat $f 1 u x$ in the base region through mechanisms (1) and (2) sufficiently to account for the amount of heat flux absorbed by the actual base region. This suggests that the dominant process in the energy balance of the base region is the absorption of heat flux by radiation.

The mechanical energy flux which passes outward through the transition region provides the energy input to the corona which balances the energy losses of the corona. Energy is removed from the corona by radiation, by the solar wind, and by inward heat conduction to the transition region. Of the order $10^{6} \mathrm{erg} \mathrm{cm}^{-2} \mathrm{sec}^{-1}$ is lost from the corona by inward heat conduction, while the combined radiative and solar-wind losses are only of the order $10^{5} \mathrm{erg} \mathrm{cm}^{-2} \mathrm{sec}^{-1}$ (Nikolsky 1969, Kopp, 1968). Therefore, the mechanical energy flux which passes through the transition region is of the order of $10^{6} \mathrm{erg} \mathrm{cm}^{-2} \mathrm{sec}^{-1}$. 
Most of the mechanical energy flux in the transition region is carried by upward propagating compression waves (Uchida, 1967). We can estimate the velocity fluctuations produced in the base region by these compression waves from the formula for the energy flux carried by onedimensional sound waves,

$$
F_{m}=\rho v^{2} a
$$

where $\rho$ is the mass density, $a$ is the sound speed, and $v$ is the. rms velocity of the oscillations due to the sound waves in the fluid. The sound speed is given by

$$
a=\left(Y \frac{k}{m} T\right)^{1 / 2},
$$

where $k$ is Boltzmann's constant, $m$ is the mean particle mass, and $\gamma$ is the ratio of specific heats. In terms of the temperature $T$ and the pressure $p=n k T$, equation (43) is

$$
F_{m}=\left(\gamma \frac{m}{k}\right)^{1 / 2} v^{2} p T^{-1 / 2} .
$$

At the base of the transition region $F_{m} \approx 10^{6} \mathrm{erg} \mathrm{cm}^{-2} \mathrm{sec}^{-1}, \mathrm{p} \approx 0.2$ dyne $\mathrm{cm}^{-2}, \mathrm{~T} \approx 10^{4} \mathrm{~K}, \mathrm{~m} \approx \mathrm{m}_{\mathrm{H}}=10^{-23.78} \mathrm{gm}$, and $\gamma \approx 5 / 3$. So, from equation (45), the rms velocity fluctuation produced in the base region by the compression waves is

$$
\mathrm{v} \approx 2.0 \times 10^{6} \mathrm{~cm} \mathrm{sec}^{-1}
$$

This estimate should be accurate to order of magnitude.

The vertical displacement $\Delta z$ resulting from the velocity oscillations of a wave of period $P$ is approximately 


$$
\Delta \mathrm{z}=\frac{1}{2} \mathrm{Pv}
$$

The compression waves which carry energy to the corona are expected to have periods of the order of $10^{2} \mathrm{sec}$ (Osterbrock, 1961). We therefore expect that vertical displacements of the order of $10^{3} \mathrm{~km}$ are produced at the base of the transition region by the upward propagating compression waves. Assuming that the compression waves are generated by the motions of the photospheric granules, the horizontal scale of the velocity fluctuations at the base of the transition should be comparable to the distance between centers of adjacent granules. The average distance between centers of adjacent granules is about $2,000 \mathrm{~km}$, which length is also representative of the horizontal dimension of vertical oscillations observed in the chromosphere (Leighton, 1963). Thus, it appears that the base region cannot be considered to be horizontally uniform on a scale of $10^{3} \mathrm{~km}$ or less.

We are now in a position to estimate the effect of the vertical oscillations on the radiative capacity of the base region through mechanism (1). That is, we may now estimate the factor by which the vertical oscillations increase the volume of the actual base region over that of the planar model base region. We have found from our model transition region that the thickness of the base region (the distance between the $10^{5} \mathrm{~K}$ level and the $10^{4} \mathrm{~K}$ level) is of the order of $10 \mathrm{~km}$. Therefore, the actual base region may be thought of as a thin surface layer coating the $10^{4} \mathrm{~K}$ "level" which is distorted into a wavy surface by the vertical displacements of the compression waves. The volume of the base region is the product of its surface area and thickness. The thickness is 
approximately inversely proportional to the heat flux entering the base region, and the heat flux entering the base region is inversely proportional to the surface area of the base region. Hence, the fractional increase $\mathrm{v}_{\mathrm{a}} / \mathrm{v}_{\mathrm{p}}$ of the volume of the actual base region over that of the planar model transition region is approximately the square of the fractional increase $A_{a} / A_{p}$ of the surface area of the actual base region over that of the planar model:

$$
\frac{\mathrm{V}_{\mathrm{a}}}{\mathrm{V}_{\mathrm{p}}} \approx\left(\frac{\mathrm{A}_{\mathrm{a}}}{\mathrm{A}_{\mathrm{p}}}\right)^{2}
$$

To estimate $A_{a} / A_{p}$, we consider the hexagonal arrangement of oscillating cells shown in Figure 12. Each +, -, or 0 marks the center of a cell. The distance between adjacent centers is assumed to be $2,000 \mathrm{~km}$. One third of the centers, those marked + , are assumed to be displaced $1,000 \mathrm{~km}$ above the mean level of the $10^{4} \mathrm{~K}$ surface; another third of the centers, marked -, are assumed to be $1,000 \mathrm{~km}$ below the mean level; and the remaining third of the centers, marked 0, are assumed to have no displacement from the mean level of the $10^{4} \mathrm{~K}$ surface. The $10^{4} \mathrm{~K}$ surface is assumed to pass smoothly through all of the cell centers. The fractional increase in the surface area of this $10^{4} \mathrm{~K}$ surface is conservatively estimated by the ratio of the area of the triangle formed by the centers of any three adjacnet + , -, and 0 cells to the projected area of this triangle on the horizontal plane. An example of such a triangle, viewed from above, is shown in Figure 12. This estimate gives

$$
\frac{A_{a}}{A_{p}}=\sqrt{2},
$$


and

$$
\frac{\mathrm{v}_{\mathrm{a}}}{\mathrm{v}_{\mathrm{p}}} \approx 2 .
$$

Thus, the non-planar nature of the actual base region may reasonably be expected to increase the radiative capacity of the actual base region over that of the planar model by a factor of 2 , which is sufficient to explain the discrepancy hetween the model and the XUV-resonance-1ine data.

We next consider the increase in the radiative capacity of the base region due to the effect of the mechanical energy flux through mechanism (2), i.e. due to the increase in the radiative power output density resulting from the presence of the mechanical energy flux. Under the approximation of collisional excitation and ionization, and radiative recombination and de-excitation, the radiative power output density is proportional to the square of the number density. Hence, we can estimate the increase in the radiative power output density by estimating the increase in the number density in the base region due to the presence of the mechanical flux.

We assume that on the average over a sufficiently long time the base region is in hydrostatic equilibrium, so that

$$
\frac{\mathrm{dp}}{\mathrm{dz}}=-\mathrm{nmg}
$$

where $\frac{d p}{d z}$ is the total pressure gradient and $n$ is the total number density. The total pressure $p$ is the sum of the thermal pressure $\mathrm{p}_{\text {th }}=\mathrm{nkT}$ and the turbulent pressure $\mathrm{p}_{\text {turb }}$ due to the velocity fluctuations in the base region. Therefore,

$$
\frac{d p}{d z}=\frac{d p_{t h}}{d z}+\frac{d p_{t u r b}}{d z}=-n m g,
$$


and we may estimate the increase in the number density $\Delta \mathrm{n}$ over what it would be if $p_{\text {turb }}$ were absent by setting

$$
\frac{d p_{\text {turb }}}{\mathrm{dz}}=-\Delta \mathrm{nmg}
$$

The gradient of the turbulent pressure may be estimated as follows. Assuming that the velocity fluctuations are isotropic, the turbulent pressure is given by

$$
\mathrm{p}_{\mathrm{turb}}=\frac{1}{3} \rho \mathrm{v}^{2}
$$

where $v$ is the rms velocity of the fluctuations. Assuming that $v$ is related to $F_{m}$ by equation (43), we have

$$
\mathrm{p}_{\text {turb }}=\frac{1}{3} \frac{\mathrm{F}_{\mathrm{m}}}{\mathrm{a}}
$$

The condition $\frac{d F}{d z}=0$, which we have assumed for the base region, then gives

$$
\frac{d p_{t u r b}}{d z}=-\frac{F_{m}}{6 a T} \frac{d T}{d z} .
$$

We may now estimate $\Delta \mathrm{n}$. From equations (53) and (56),

$$
\Delta \mathrm{n}=\frac{\mathrm{F}_{\mathrm{m}}}{6 \mathrm{mgaT}} \frac{\mathrm{dT}}{\mathrm{dz}}
$$

Here,

$$
\frac{\mathrm{dT}}{\mathrm{dz}} \approx \frac{\Delta \mathrm{T}}{\Delta \mathrm{z}}
$$

where $\Delta \mathrm{T}$ is the temperature change in the base region $\left(\Delta \mathrm{T} \approx 10^{5} \mathrm{~K}\right)$ and $\Delta z$ is the vertical extent of the region of the atmosphere in which this temperature change occurs. Due to the vertical displacements 
of the upward propagating compression waves, $\Delta z$ is of the order of $10^{3} \mathrm{~km}$ for the temperature range of $10^{4} \mathrm{~K}$ to $10^{5} \mathrm{~K}$. Adopting $\Delta \mathrm{z}=$ $10^{3} \mathrm{~km}, \Delta \mathrm{T}=\mathrm{T}=10^{5} \mathrm{~K}$, and $\mathrm{F}_{\mathrm{m}}=10^{6} \mathrm{erg} \mathrm{cm}^{-2} \mathrm{sec}^{-1}$ in equations (57) and (58), we obtain

$$
\Delta \mathrm{n} \approx 1.4 \times 10^{10} \mathrm{~cm}^{-3}
$$

This estimate is accurate only to order of magnitude, but it indicates that the increase in the number density in the base region due to the turbulent pressure is of the same order as the density in our model base region $\left(\mathrm{n} \approx 10^{10} \mathrm{~cm}^{-3}\right.$ at $\mathrm{T}=10^{5} \mathrm{~K}$ ) which has no turbulent pressure. Therefore, it appears that the presence of the velocity fluctuations could easily increase the radiative capacity of the base region as much through density increase (mechanism (2)) as through volume increase (mechanism (1)). Again, this suggests that most of the heat flux entering the base region is converted into radiation rather than into mechanical energy flux. 


\section{ACKNOWLEDGEMENTS}

We would like to thank Professor P. A. Sturrock for patient guidance and advice, and we thank him as well as Dr. E. Tademaru, Dr. D. W. Goldsmith, and Mr. G. C. Dijkhuis for stimulating and helpful discussions during the course of the work reported here. This work was supported in part by the National Aeronautics and Space Administration under Grant NGL 05-020-272 and in part by the Air Force Office of Scientific Research under Contract F44620-69-C-0008. 
Allen, C.W.: 1963, Astrophysical Quantities, Athlone Press, p. 185. Allen, C.W.: 1965, Space Sci.Rev. 4, 91. Athay, R. G.: 1966, Astrophys. J. 145, 784 .

Athay, R. G.: 1969, Solar Phys. 2, 51. Beckers, J. M.: 1968, Solar Phys. 3 , 367.

Cox, D. P. and Tucker, W. H.: 1969, Astrophys. J. 157, 1157.

Delcroix, A. and Lemaire, A.: 1969, Astrophys. J. 156, 787 .

Devoto, R. S.: 1968, J. Plasma Phys. 2, 617.

Dupree, A. K. and Goldberg, L.: 1967, Solar Phys. 1, 229.

Goldberg, L., Müller, E. and Aller, L.: 1960, Astrophys. J. Supp1 . $\underline{5}, 1$.

Kopp, R. A.: 1968, Air Force Cambridge Research Laboratories Scientific Report No. 4, AFRCL-68-0312.

Kopp, R. A. and Kuperus, M.: 1968, Solar Phys. ㄴ, 212.

Kuperus, M. and Athay, R. G.: 1967, Solar Phys. 1, 361.

Leighton, R. B.: 1963, Ann. Rev. Astron. and Astrophys. 1, 19.

Nikolsky, G. M.: 1969, Solar Phys. 6, 399.

Noyes, R. W., Withbroe, G. L. and Kirschner, R. P.: 1970, Solar Phys. $\underline{11}, 388$.

Osterbrock, D. E.: 1961, Astrophys. J. 134, 347.

Pottasch, S. R.: 1964, Space Sci. Rev. 3, 816.

Suemoto, Z. and Hiei, E.: 1962, Publ. Astron. Soc. Japan 14, 33.

Uchida, Y。: 1967, Ast rophys.J.147, 181.

Vincenti, W. G. and Kruger, C. H.: 1965, Introduction to Physical Gas Dynamics, John Wiley and Sons, Inc., p. 18.

Withbroe, G. L.: 1970, Solar Phys, 11, 42. 


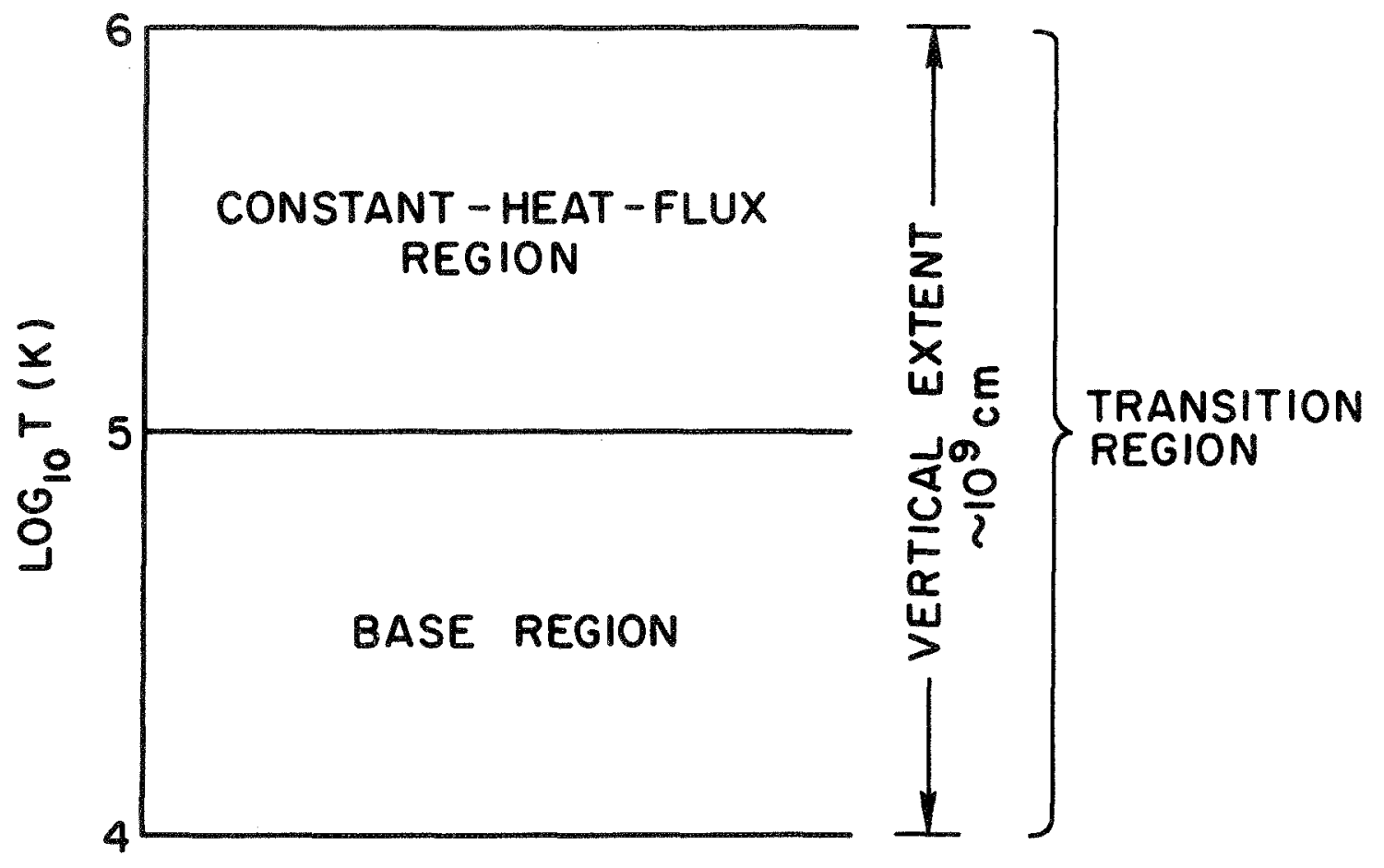

Figure 1. Schematic representation of the transition region. 


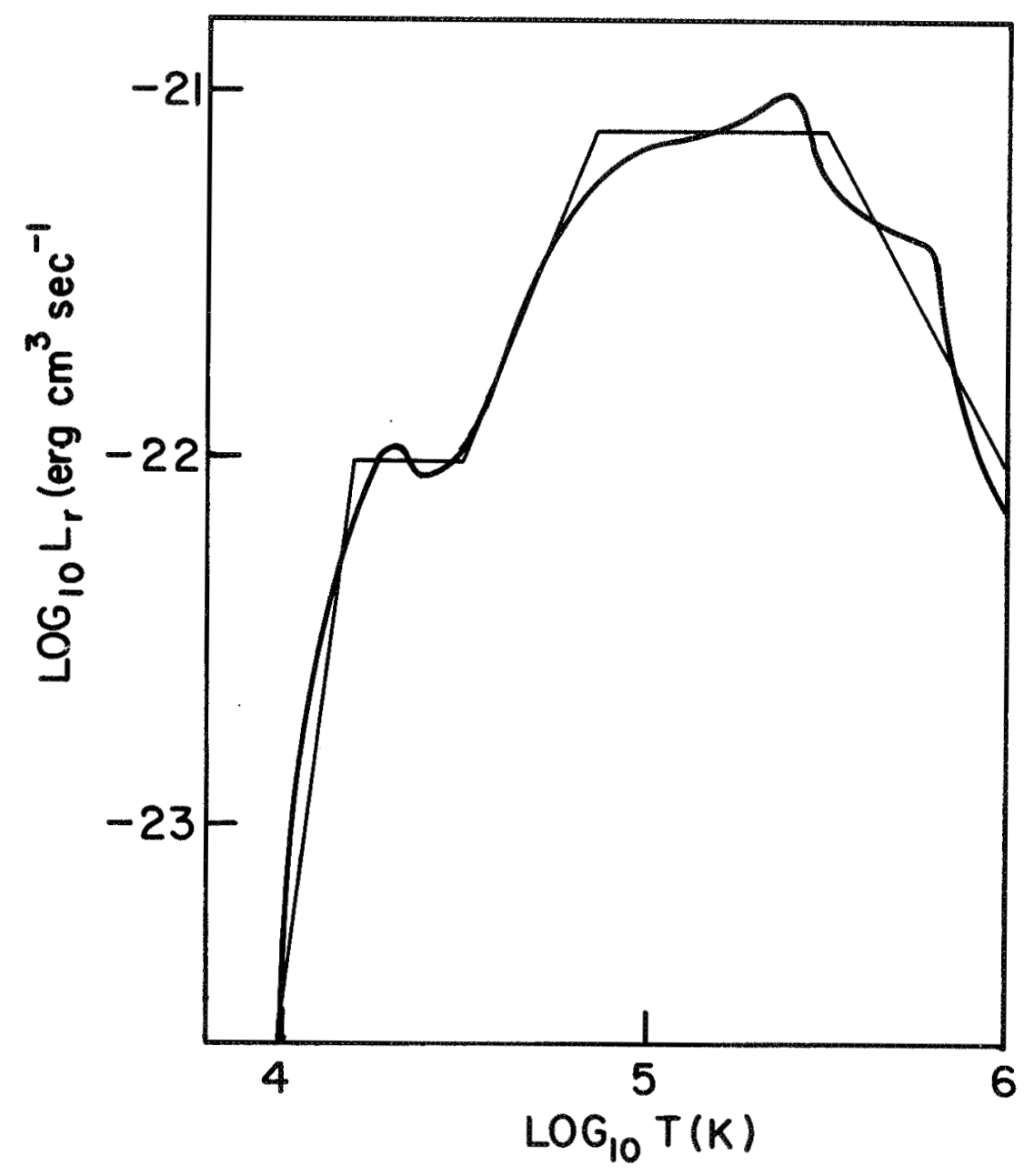

Figure 2. Radiative cooling coefficient curve computed by Cox and Tucker (1969) and the straight-line-segment fit adopted for our model. 


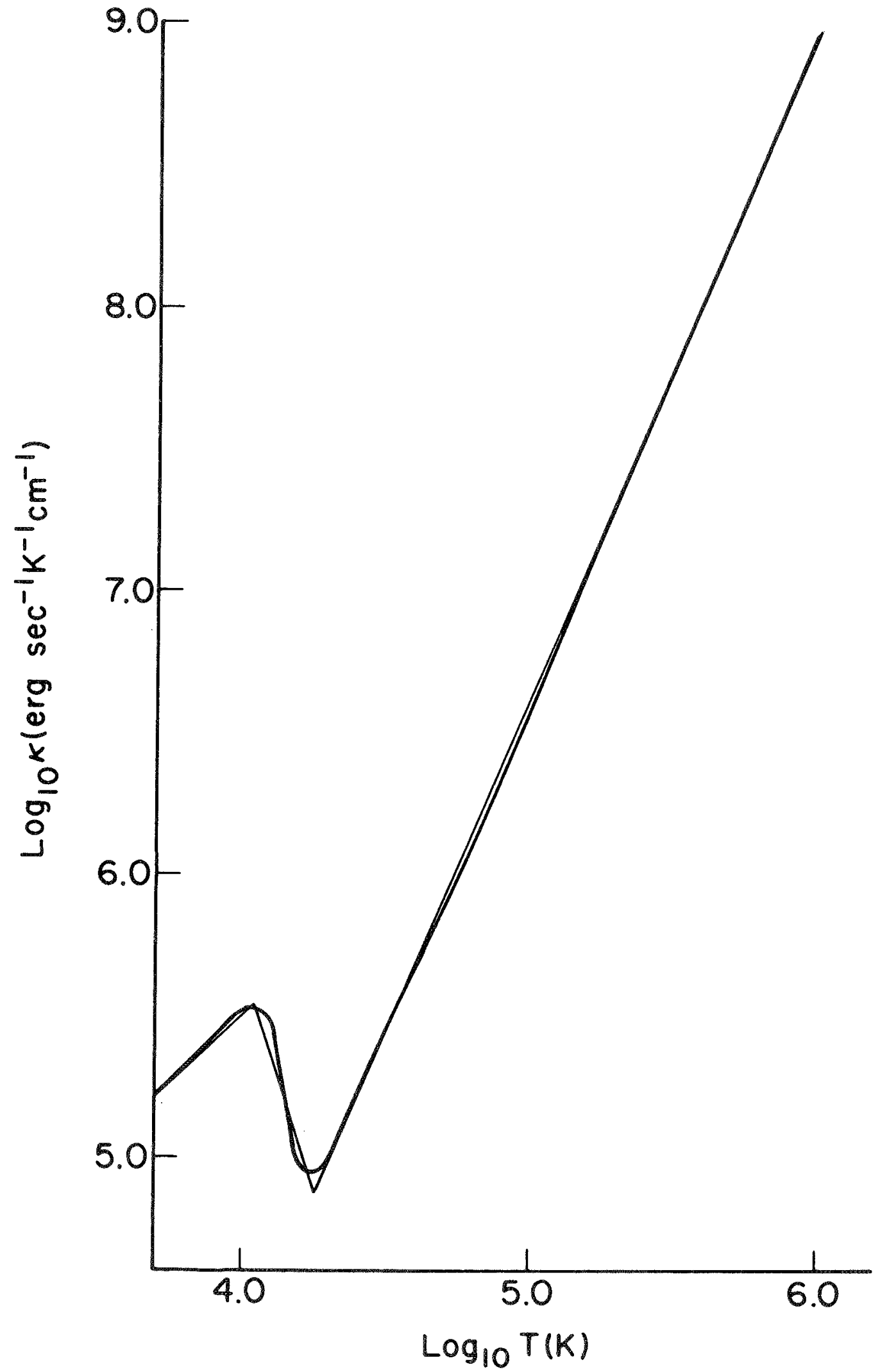

Figure 3. Thermal conductivity curve derived from the results of Devoto (1968) and Delcroix and Lemaire (1969) and the straight line fit adopted for our model. 


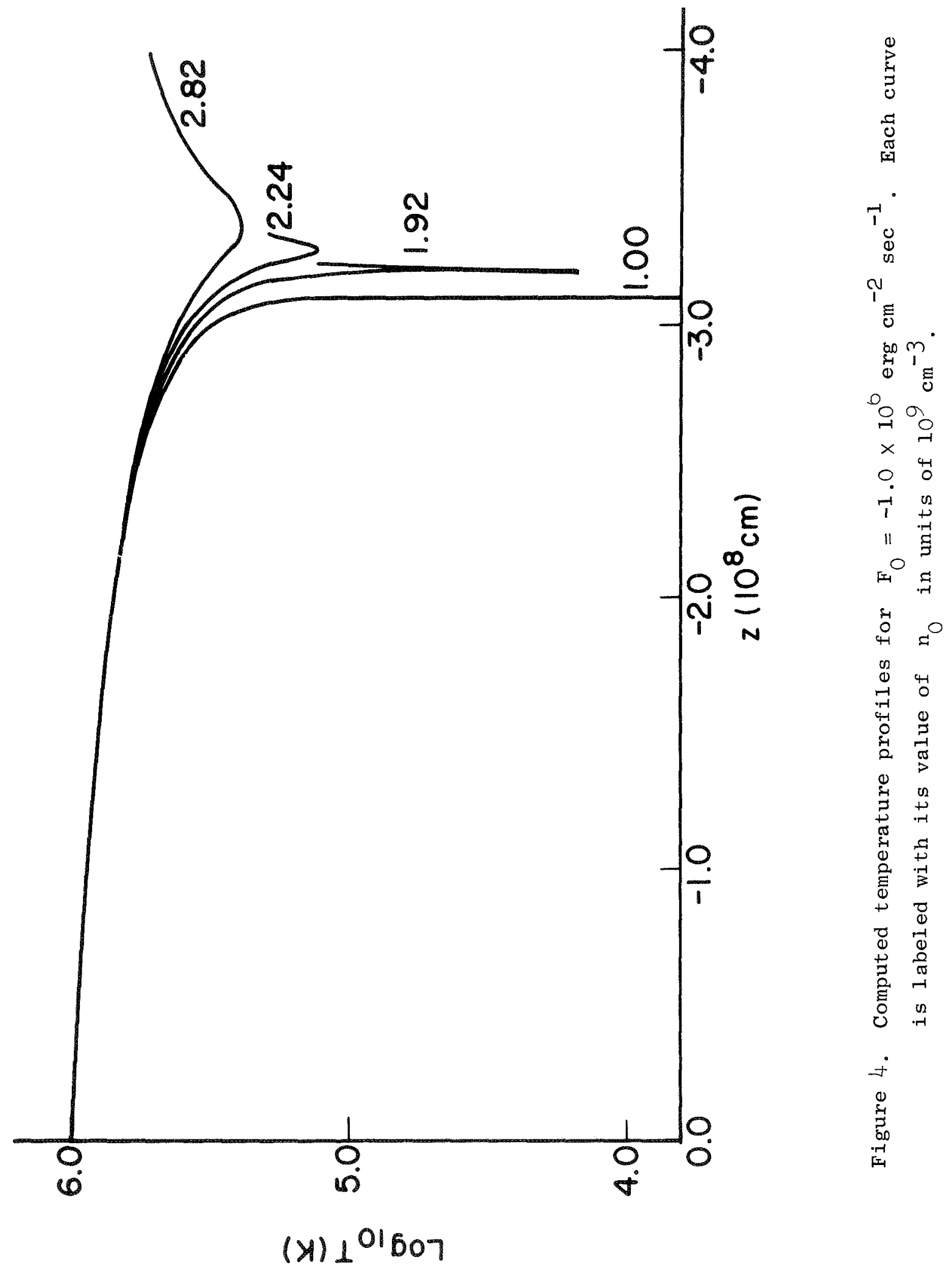




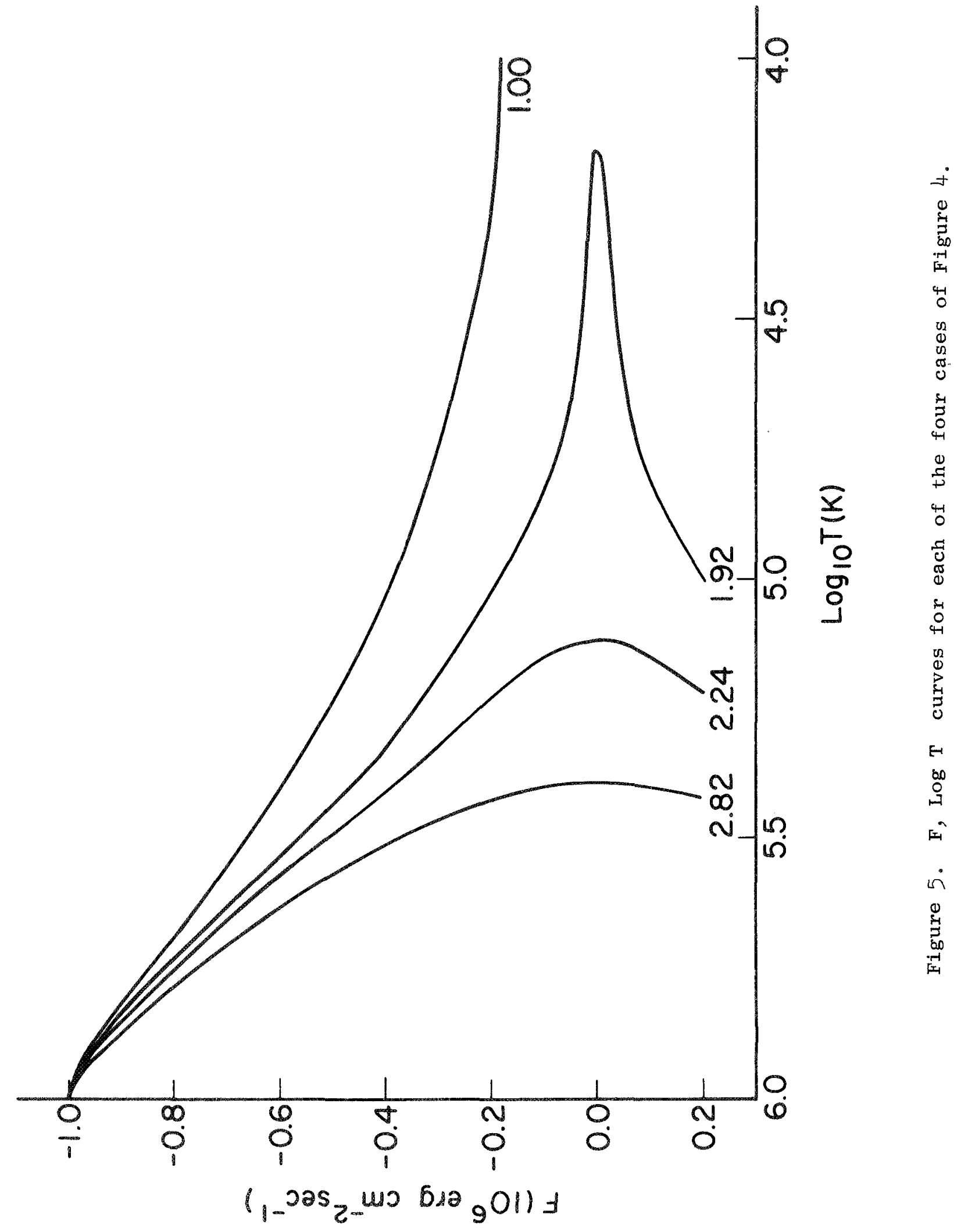




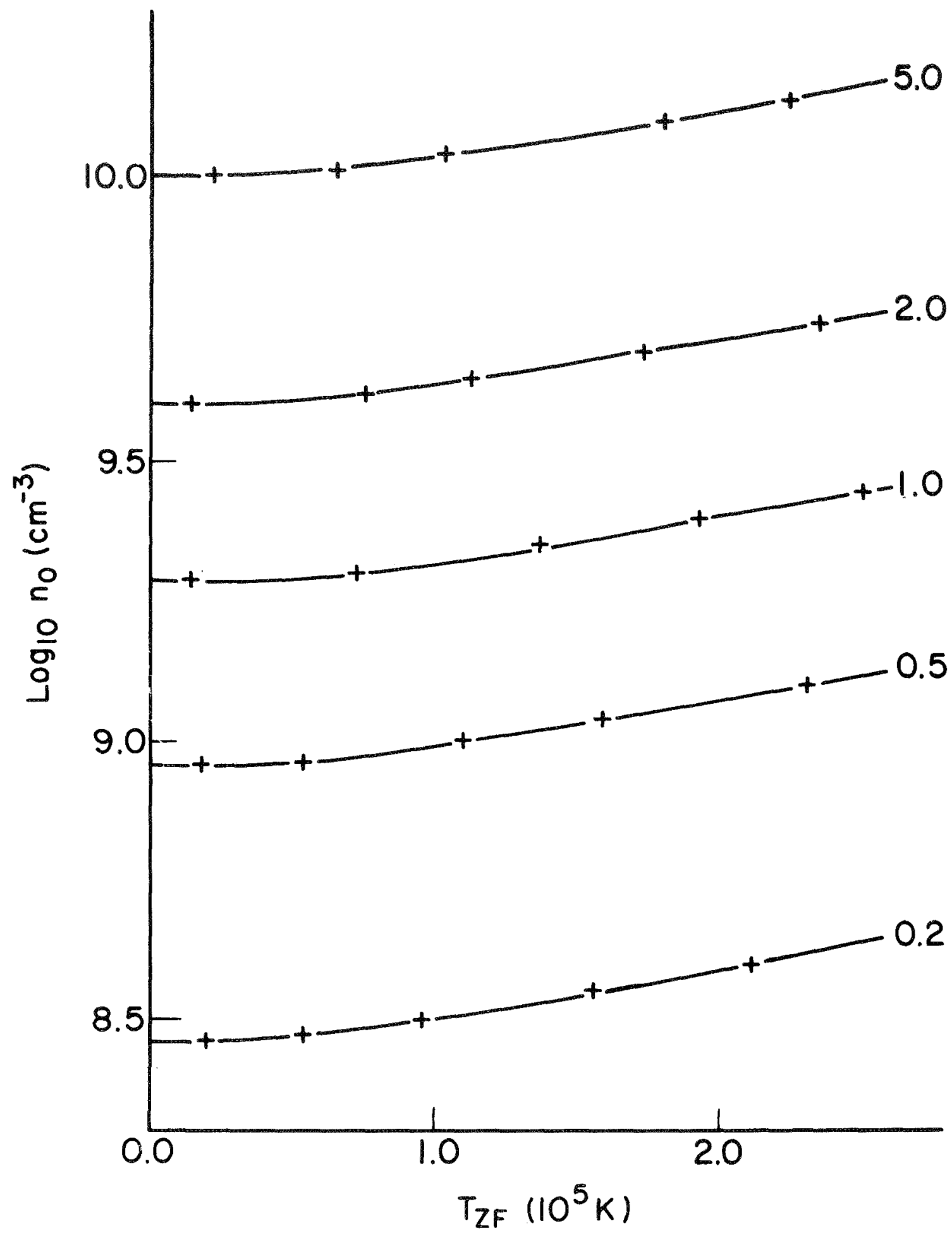

Figure 6. Curves of Log $n_{0}$ VS $T_{\text {ZF }}$ for constant values of $F_{O}$. Each curve is labeled with its value of $-F_{O}$ in units of $10^{6} \mathrm{erg} \mathrm{cm}^{-2} \mathrm{sec}^{-1}$. 


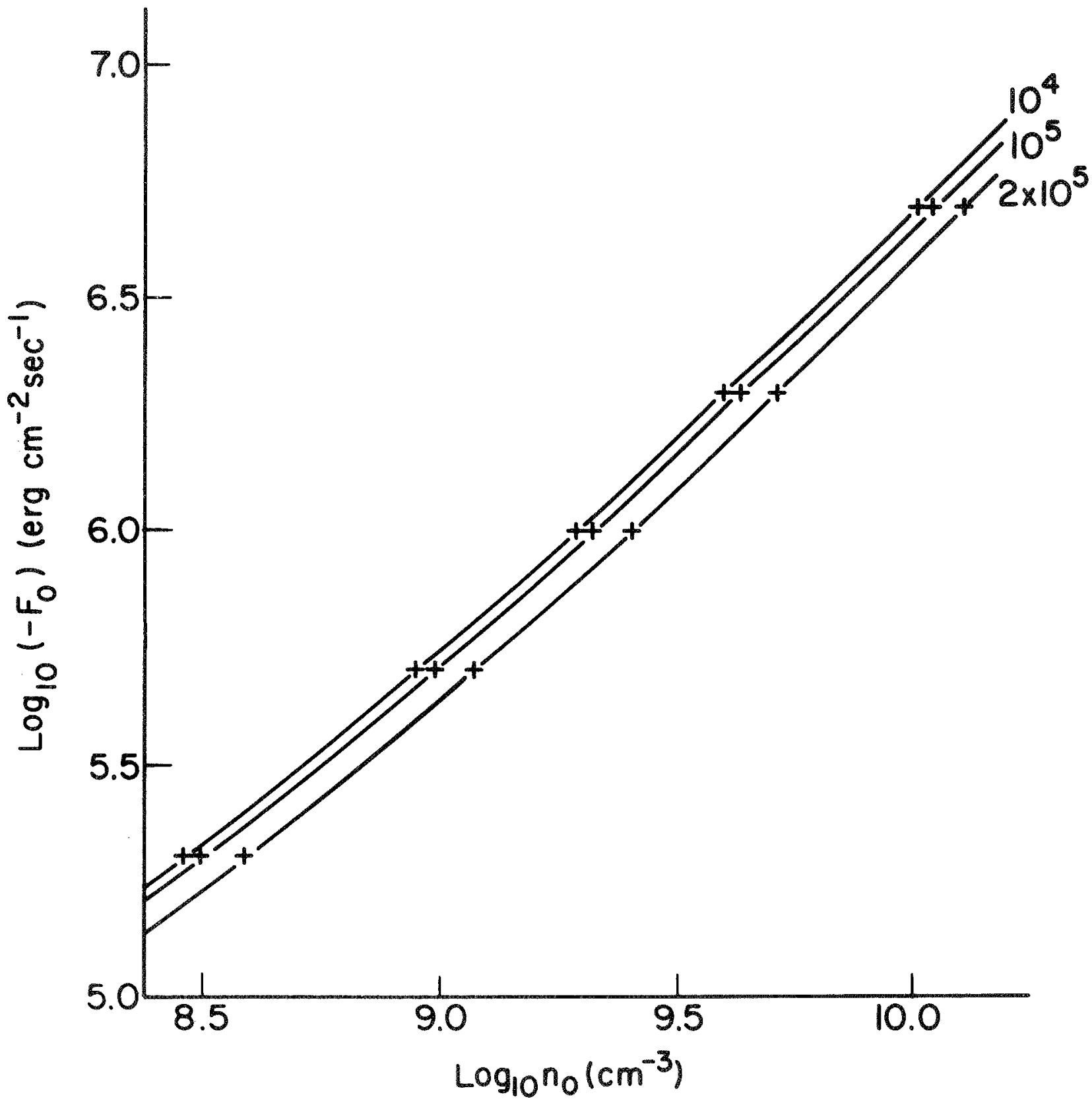

Figure 7. $\log \left(-\mathrm{F}_{\mathrm{O}}\right), \log \mathrm{n}_{\mathrm{O}}$ curves for constant values of $\mathrm{T}_{\mathrm{ZF}}$. Each curve is labeled with its value of $\mathrm{T}_{\mathrm{ZF}}$ in units of degrees $\mathrm{K}$. 


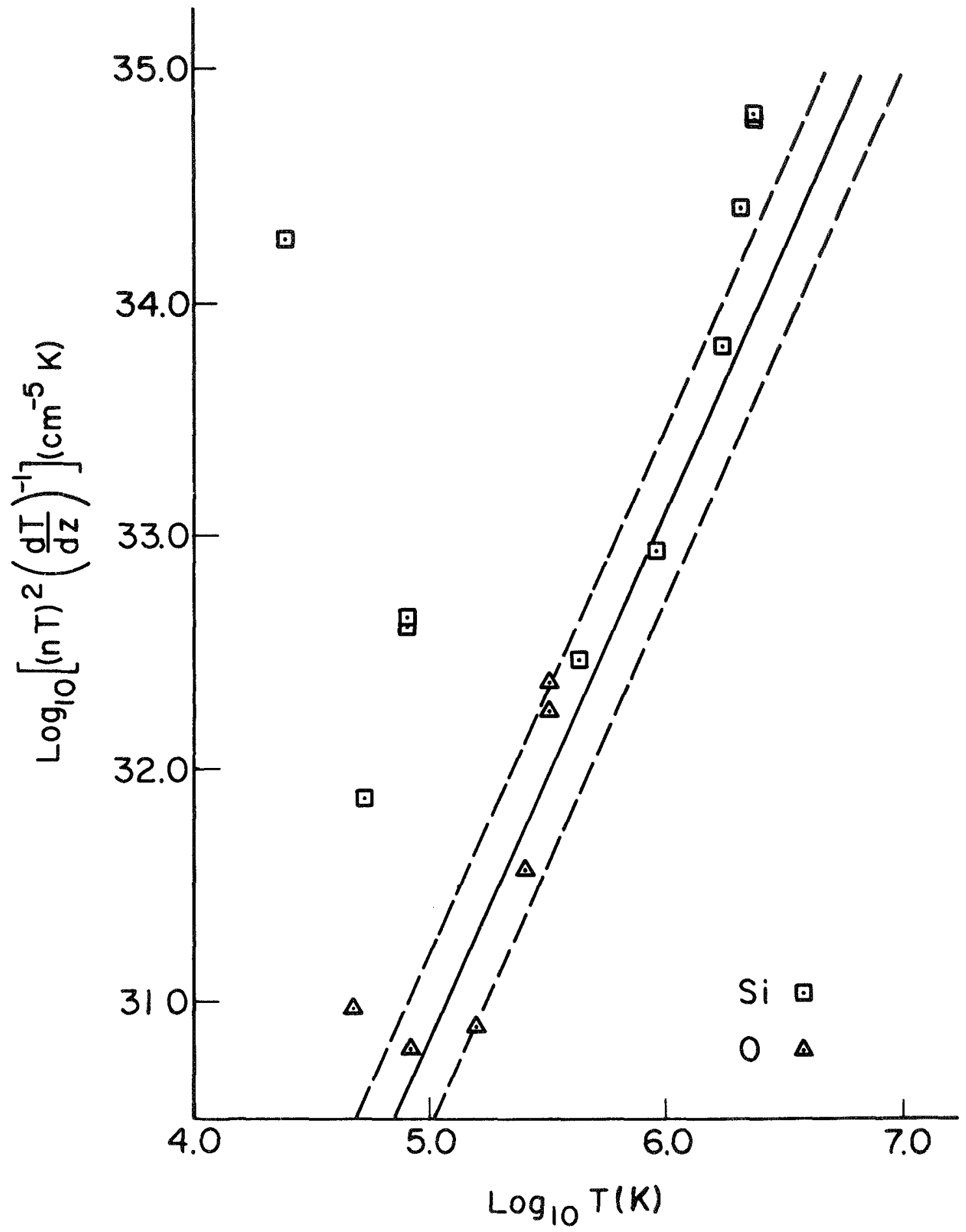

Figure 8. Reduced XUV-resonance-1ine data drom Dupree and Goldberg (1967). In the temperature range $10^{5} \mathrm{~K}$ to $10^{6} \mathrm{~K}$, the straight lines correspond to constant downward heat flux. The dashed lines show that the data scatter around the "best-fit" solid line by a factor of two. 


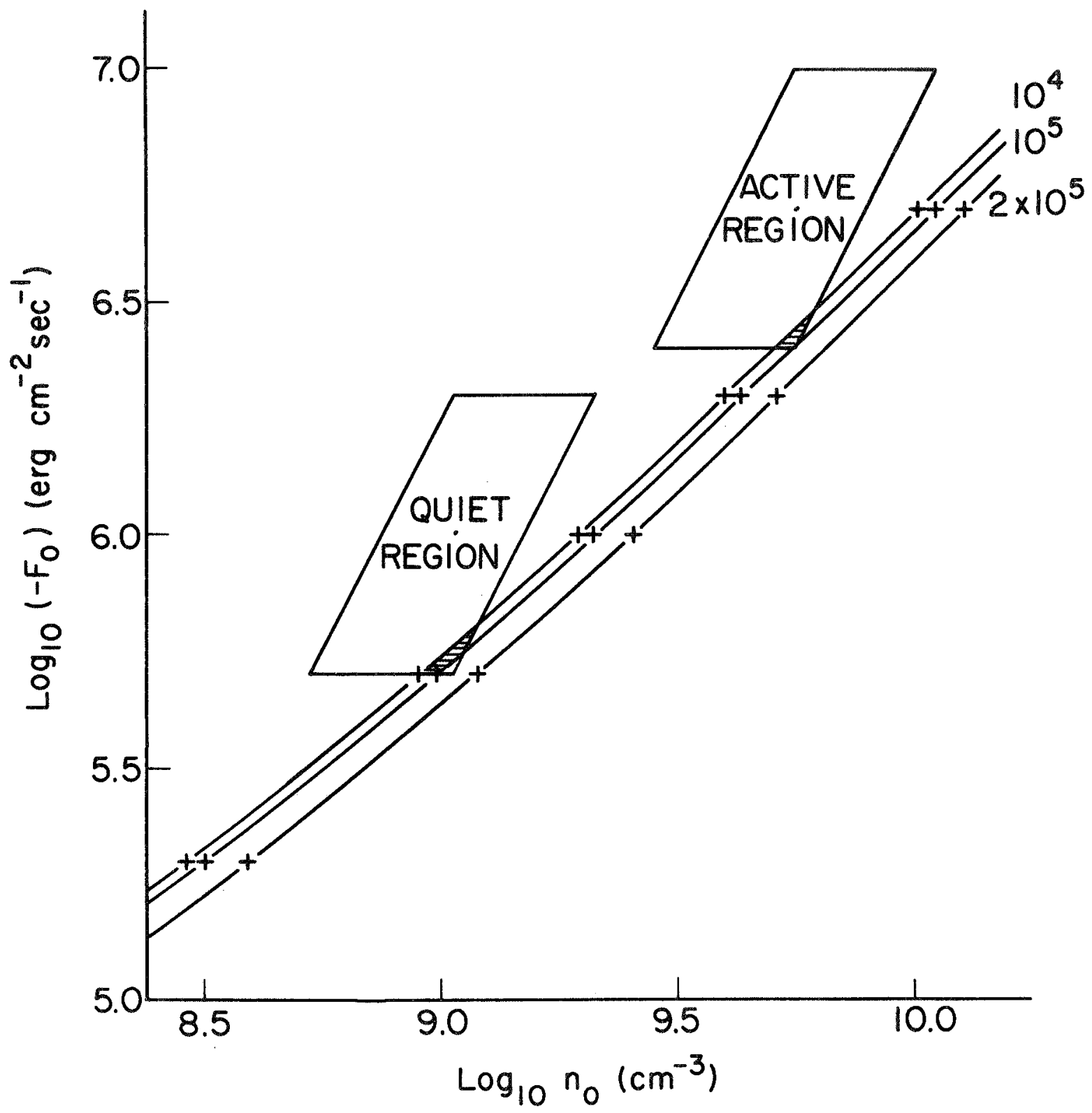

Figure 9. Comparison of the model with the requirements of the XUV-resonance-line data. In the shaded regions the model is compatible with the data. 


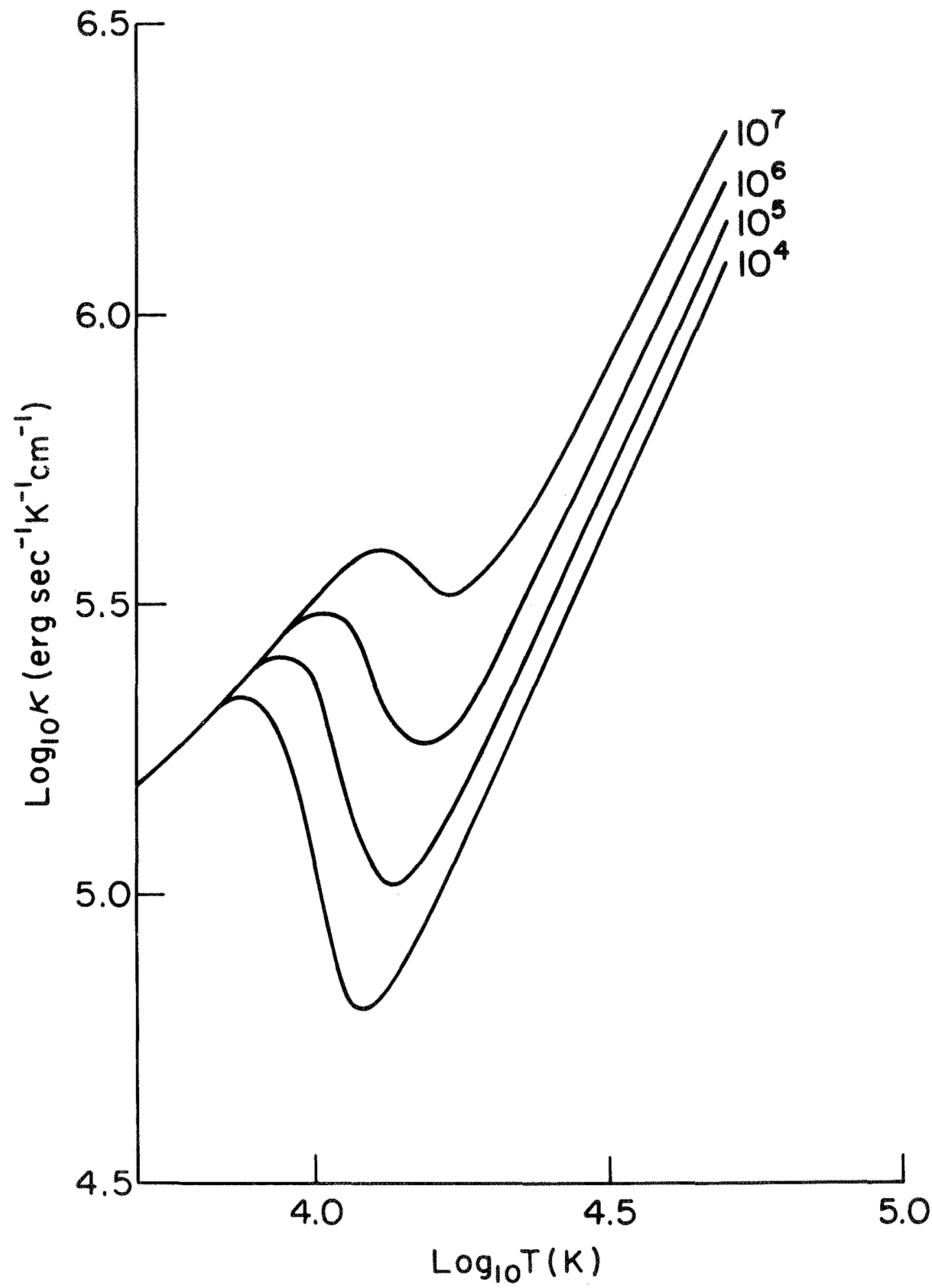

Figure 10. Conductivity curves obtained from Devoto's (1968) table for partially ionized hydrogen at constant pressure. Each curve is labeled with its value of the pressure in units of 1.013 dyne $\mathrm{cm}^{-2}$. 


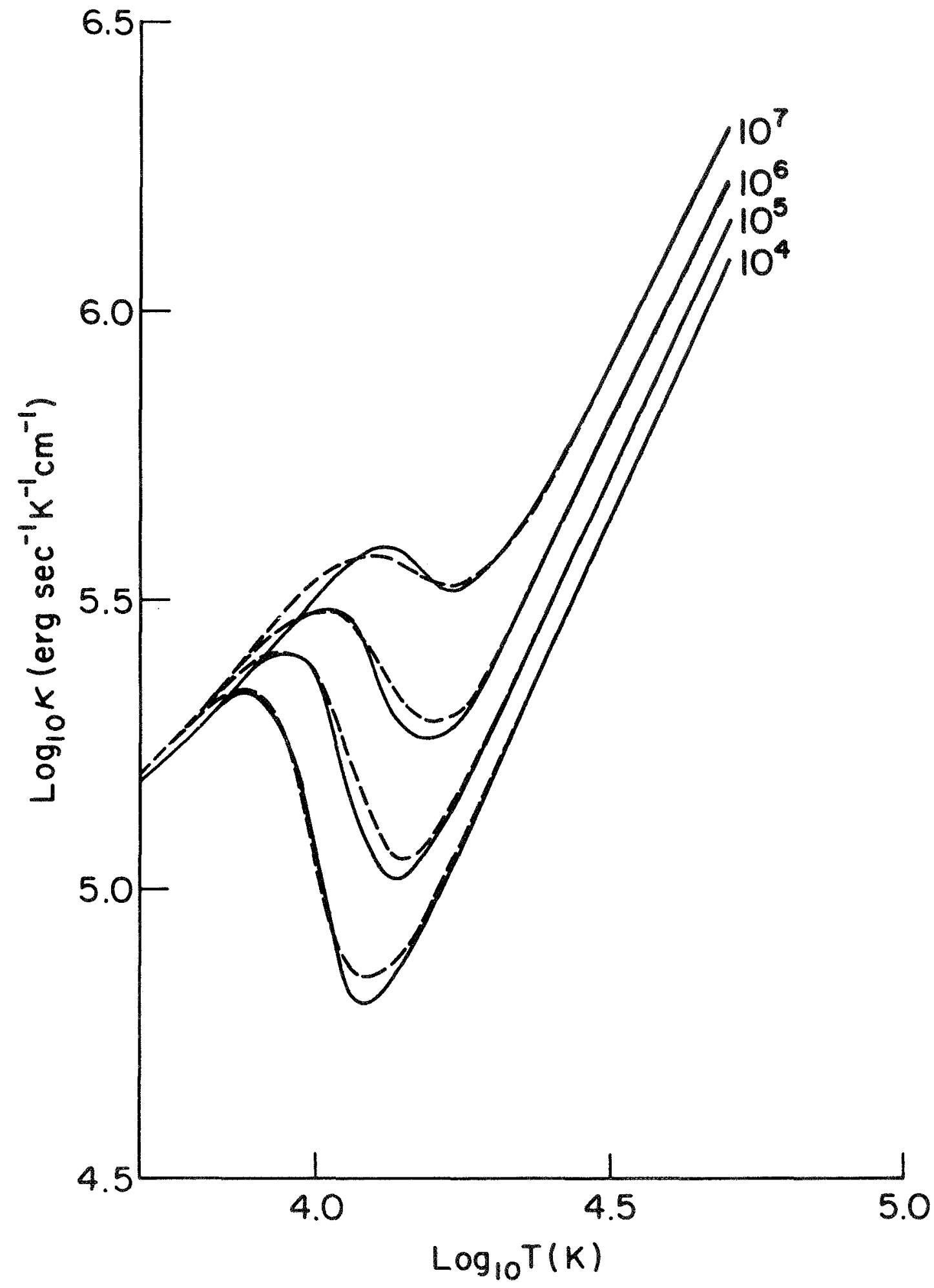

Figure 11. Comparison of the conductivity given by equation (35)

(dashed curves) with Devoto's conductivity (solid curves)

for $\sigma_{\mathrm{HH}}=9.12 \times 10^{-14} \mathrm{~T}^{-1 / 2} \mathrm{~cm}^{2}$ and $\sigma_{\mathrm{Hp}}=7.95 \times$ $10^{-11} \mathrm{~T}^{-1} \mathrm{~cm}^{2}$. 


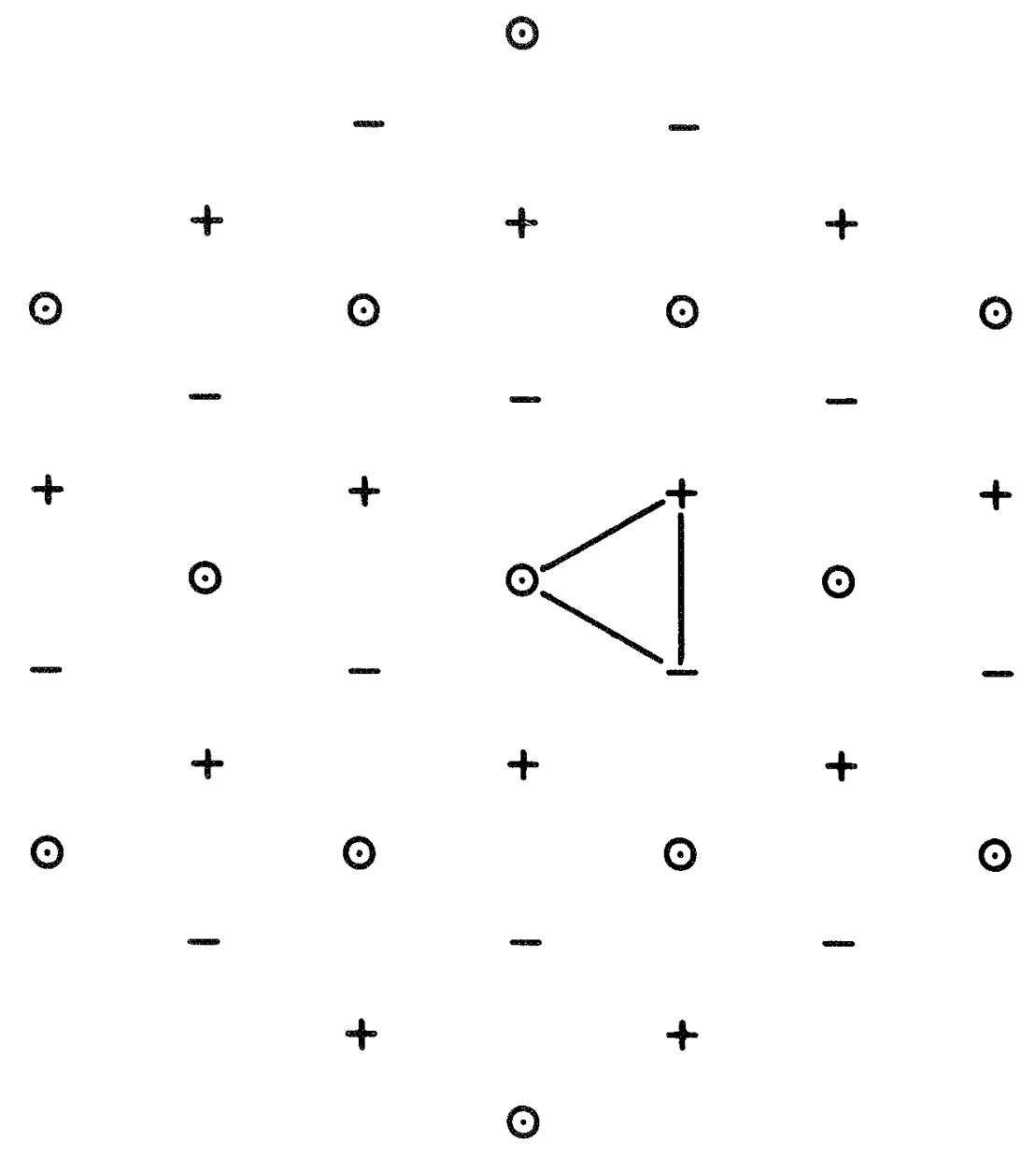

Figure 12. Configuration of centers of oscillating cells adopted to estimate the increase in the surface area of the $10^{4} \mathrm{~K}$ level due to the vertical displacements of the oscillations. 